\title{
Conexões
}

\section{Qualidade de vida e autoconceito de atletas paranaenses de basquetebol em cadeira de rodas}

Fernanda Gimenez Milani ${ }^{1}$ Andressa Ribeiro Contreira ${ }^{1}$ Vinicius Machado de Oliveira ${ }^{1}$ Aryelle Malheiros Caruzzo ${ }^{1}$

\section{RESUMO}

Este estudo objetivou investigar a qualidade de vida e o autoconceito de atletas paranaenses de basquetebol em cadeira de rodas. Fizeram parte do estudo atletas, do sexo masculino, participantes da $2^{\mathrm{a}}$ etapa do Paranaense de Basquete em Cadeira de Rodas, sediada na cidade de Maringá-PR. Para a coleta de dados foram utilizados o questionário Medical outcomes study 36 - item short - form health survey - SF-36 (CICONELLI, 1997) e a Escala Fatorial de Autoconceito - EFA (TAMAYO, 1981). Para análise dos dados, foram utilizados os testes Kolmogorov-Smirnov, Mann-Whitney e coeficiente de correlação de Spearman, adotando-se $(P \leq 0,05)$. Os resultados evidenciaram que a maioria dos atletas possui um alto nível de autoconceito e bom nível de qualidade de vida. Na comparação entre a percepção da qualidade de vida conforme a classificação do autoconceito os atletas com alto nível de autocontrole apresentaram maior score de qualidade de vida para a dimensão "limitação por aspectos físicos", sendo esse valor significativamente maior quando comparados aos atletas de nível baixo/médio $(\mathrm{p}=0,029 *)$. Não foram encontradas correlações fortes entre as dimensões da qualidade de vida e autoconceito. Atletas de paradesporto da modalidade de basquetebol de cadeira de rodas apresentaram bons níveis de qualidade de vida e autoconceito.

Palavras-chave: Qualidade de vida. Autoconceito. Deficiência física. Esporte adaptado.

\footnotetext{
${ }^{1}$ Universidade Estadual de Maringá

Recebido em: 13 abr. 2017

Aprovado em: 9 out. 2018

Contato: fernandagmilani@gmail.com
} 


\section{Quality of life and self-concept of paranian athletes of basketball in wheelchair}

\section{Abstract}

The objective of this study was to investigate the quality of life and the self-concept of athletes from the state of Paraná in wheelchair basketball. The study included athletes, male, participants of the second stage of the Paranaense Basketball in Wheelchair, headquartered in the city of Maringá-PR. For the data collection, the Medical outcomes study questionnaire 36 - item short - form health survey - SF-36 (CICONELLI, 1997) and the Factorial Scale of Self-concept - EFA (TAMAYO, 1981) were used. For data analysis, the Kolmogorov-Smirnov, Mann-Whitney and Spearman correlation coefficients were used, adopting $(\mathrm{P} \leq 0.05)$. The results showed that most athletes have a high level of self-concept and a good level of quality of life. In the comparison between the perception of the quality of life according to the classification of the self-concept, the athletes with a high level of selfcontrol presented a higher score of quality of life for the dimension "limitation by physical aspects", being this value significantly higher when compared to low level athletes / Median $\left(\mathrm{p}=0.029^{*}\right)$. No strong correlations were found between the dimensions of quality of life and self-concept. Wheelchair basketball paradesport athletes presented good levels of quality of life and self-concept.

Keywords: Quality of life. Self Concept. Physical Disabilities. Adapted sports.

\section{Calidad de vida y autoconcepto de atletas de baloncesto en silla de ruedas en el Paraná}

\section{RESUMEN}

Este estudio tuvo como objetivo investigar la calidad de vida y el autoconcepto de atletas paranaenses de baloncesto en silla de ruedas. Los participantes del estudio, atletas masculinos de la $2^{\text {a }}$ Etapa de Baloncesto de Silla de Ruedas Paranaense, con sede en la ciudad de Maringá-PR. Para la recolección de datos se utilizó el cuestionario Medical outcomes study 36 - ítem short - form health survey - SF36 (CICONELLI, 1997) y la Escala Factorial de Autoconcepto - EFA (TAMAYO, 1981). Para el análisis de datos, se utilizaron las pruebas de Kolmogorov-Smirnov, Mann-Whitney y el coeficiente de correlación de Spearman, adoptando $(P \leq 0,05)$. Los resultados mostraron que la mayoría de los atletas tiene un alto nivel de autoconcepto y buen nivel de calidad de vida. En la comparación de la percepción de la calidad de vida de acuerdo a la clasificación de auto-atletas con un alto nivel de autocontrol tuvieron mayor puntuación de la calidad de vida de la dimensión "limitaciones debido a los aspectos físicos", siendo significativamente mayor en comparación con los atletas de bajo o medio nivel / Avg ( $\left.\mathrm{p}=0,029^{*}\right)$. No se encontraron fuertes correlaciones entre las dimensiones de la calidad de vida y el concepto de sí mismo. atletas parasports de modo de baloncesto en silla de ruedas mostraron buenos niveles de calidad de vida y autoconcepto.

Palabras Clave: Calidad de vida. Ser. Deficiencia física. Deporte Adaptado. 


\section{INTRODUÇÃO}

A expectativa de vida em países em desenvolvimento como o Brasil vem crescendo consideravelmente, fruto dos avanços da medicina, das melhorias nas condições ambientais e sociais que têm possibilitado um tempo de sobrevida maior para a população (MARTINS; DASCAL; MARQUES, 2013; OLIVEIRA, 2016). Contudo, não basta apenas viver muito é necessário também viver bem. De acordo com Araújo e Araújo (2000), para viver bem é preciso que todas as pessoas possam alcançar níveis dignos de sobrevivência, bem como ter acesso aos avanços científicos e ter seus direitos humanos preservados. Uma vida fisicamente ativa favorece esse advento, e se agregado a outras medidas pode refletir positivamente na vida dos seres humanos. Assim, possuir níveis adequados de atividade física é um dos prérequisitos essenciais para apresentar um estilo de vida considerado saudável.

Entretanto, a atividade física possui as suas limitações, já que podem ser atividades mais simples e sem a devida intencionalidade. Dessa forma, o exercício físico é recomendado, tendo em vista que proporciona atividades mais estruturadas que correspondem melhor às especificidades desejadas. O exercício físico pode ser entendido como um movimento humano que possui objetivos prático-pedagógicos, necessitando ser uma ação desejada e operacionalizada pelos sistemas e aparelhos corporais humanos, se apresentando como um dos pilares desse conjunto. Dessa forma, movimentos presentes na dança, luta, ginástica, jogos entre outros são considerados exercícios e colaboram para uma vida saudável (MADUREIRA; FONSECA; MAIA, 2003).

Estudos epidemiológicos apontam que a adesão à prática de exercícios físicos regulares caracterizados por níveis desejados de aptidão física está relacionada a menores taxas de mortalidade e melhor qualidade de vida na medida em que facilitam a locomoção, adequam a composição corporal e aumentam a disposição para as tarefas diárias (BLAIR et al., 1995; PAFFENBARGER, 1994). Além dos benefícios biológicos, desfechos positivos na esfera psicológica também têm sido observados, destacando-se a melhora do humor e o aumento do autoconceito (TAMAYO; 2001; ZUCHETTO; CASTRO, 2002; LATORREROMÁN; MARTÍNEZ; GARCÍA-PINILLOS, 2015).

Em se tratando de pessoas com deficiência, os exercícios físicos possuem benefícios ainda mais perceptíveis, pois além de prover melhorias nas capacidades funcionais, também apresentam grande participação no desenvolvimento biopsicossocial do indivíduo, promovendo uma melhor qualidade de vida (ZUCHETTO; CASTRO, 2002). É em função dessa prerrogativa, que os esportes adaptados têm se tornado parte integrante da vida de muitas pessoas com deficiência, pois além de ajudar no processo de reabilitação e melhora das capacidades, tem papel fundamental na sociabilização e na melhora da autoestima. Segundo o Instituto Brasileiro dos Direitos a Pessoas Com Deficiência - IBDD (2008, p. 23), o esporte é utilizado como um recurso para reabilitação, integração social e conscientização da sociedade sobre o potencial da pessoa com deficiência, indicando a relevância da prática esportiva para esta população. 
Com relação ao autoconceito, Gallahue e Ozmun (2001) definem como a percepção do indivíduo a respeito de suas características, atributos e limitações, sendo similares ou diferentes das outras pessoas. Além disso, a autoestima é considerada como um componente que determina essa variável, indicando de forma consciente ou inconsciente, quem ou o que achamos que somos (BRANDEN, 2000). Nessa perspectiva estes conceitos remetem à definição de Tamayo (1993) adotada pelo presente estudo, que define o autoconceito como a estrutura cognitiva que organiza as experiências passadas do indivíduo, reais ou imaginárias, controla o processo informativo relacionado consigo mesmo e exerce uma função de autorregularão.

Assim, conhecendo a importância da prática regular de atividade física/exercício físico para as pessoas com deficiência física, sobretudo no que tange à esfera psicológica e sua emergente área de investigação (ZUCHETTO; CASTRO, 2000, MARTINS; RABELO, 2008, NOCE et al., 2009), o presente estudo teve como objetivo, investigar a percepção de qualidade de vida e o nível de autoconceito em atletas da modalidade de basquetebol em cadeira de rodas, buscando especificamente comparar a percepção da qualidade de vida dos atletas de basquete em cadeira de rodas considerando a dimensão autocontrole do autoconceito e correlacionar a qualidade de vida e o autoconceito dos atletas.

\section{MÉTodo}

\section{Amostra}

Fizeram parte do estudo 55 atletas deficientes físicos do sexo masculino com média de idade de 34,73 ( \pm 8,54 anos), participantes da $2^{\text {a }}$ etapa da Competição Paranaense de Basquete em cadeiras de rodas, sediada na cidade de Maringá. Os atletas eram integrantes de 7 equipes das cidades de Cascavel, Guarapuava, Foz do Iguaçu, Curitiba, Maringá e Ponta Grossa.

As características dos participantes do estudo quanto ao tipo e etiologia da deficiência são apresentadas na Tabela 1.

Tabela 1 - Distribuição de frequência e percentual dos atletas de basquete em cadeira de rodas quanto ao tipo e etiologia da deficiência

\begin{tabular}{lcc}
\hline Tipo de deficiência & F & \% \\
\hline Congênita & 9 & 16,4 \\
Adquirida & 46 & 83,6 \\
Total & 55 & 100 \\
\hline Etiologia da Deficiência & F & \% \\
\hline Violência Urbana & 30 & 54,5 \\
Relacionadas à Saúde & 25 & 45,5 \\
Total & 55 & 100 \\
\hline
\end{tabular}

A coleta de dados deu-se mediante a assinatura do termo de consentimento livre 
esclarecido. Após a sinalização de consentimento, os atletas receberam os questionários para o preenchimento, sendo esses aplicados nos períodos em que as esquipes não estavam jogando. O estudo foi aprovado pelo Comitê de Ética em Pesquisa da Universidade Estadual de Maringá (UEM) sob parecer nº 339/2011.

Para a análise da qualidade de vida foi utilizado Medical Outcomes Study 36 (item short - Form Health Survey - SF-36) criado a partir de uma revisão dos instrumentos ligados à qualidade de vida já existente na literatura nos últimos 20 anos (CICONELLI 1997). Esse instrumento avalia 8 aspectos distintos: capacidade funcional (10 itens); limitação por aspectos físicos (4 itens); limitação por aspectos emocionais (3 itens); dor (2 itens); estado geral de saúde (5 itens); vitalidade (4itens); aspectos sociais ( 2 itens) e saúde mental (5 itens). As questões possuem escala Likert que variam de 1 a 7 pontos, podendo atingir um score entre 0 a 100 pontos. Para a categorização dos dados, os resultados foram divididos em: regular $(0$ - 30 pontos $)$, boa $(31$ - 60 pontos $)$ e muito boa qualidade de vida $(61-100$ pontos).

O autoconceito foi avaliado por meio da Escala Fatorial de Autoconceito - EFA (TAMAYO, 1981). A escala é composta por 51 questões que contém 2 adjetivos opostos cada, dos quais o indivíduo deve marcar um valor entre 1 e 7 , devendo analisar quais dos adjetivos entre "se aplica muito" a "se aplica pouco" ou os dois adjetivos se aplicam igualmente ao indivíduo. A Escala é dividida em seis categorias atitude social, segurança, autocontrole, ético-moral, somático, receptividade social. A pontuação é feita somando os itens referentes a cada subescala, sendo que quanto maiores os valores, maior é o autoconceito do respondente para aquela subescala. Para a classificação os resultados foram organizados em: baixo $(0,0-3,0$ pontos), médio ( $3,1-5,5$ pontos) e alto autoconceito (5,6 - 7,0 pontos). Devido à baixa frequência de atletas na categoria "baixo autoconceito", foram estabelecidas as seguintes categorias baixo/médio ( $0,0-5,5$ pontos) e alto $(5,6-7,0$ pontos). Estes critérios foram estabelecidos para a análise da comparação.

\section{ANÁLISE DE DADOS}

Os dados foram analisados no pacote estatístico SPSS versão 20.0. Para verificação da normalidade dos dados foi utilizado o teste Kolmogorov Smirnov, sendo identificada distribuição não-normal, dessa forma, os resultados foram analisados com a utilização de testes não-paramétricos. Para as análises descritivas os atletas foram classificados quanto aos níveis de autoconceito e categorizados em três níveis, segundo a pontuação na Escala de Autoconceito: baixo ( $0,0-3,0$ pontos), médio ( $3,1-5,5$ pontos) e alto $(5,6-7,0$ pontos). Os critérios estabelecidos para as análises de comparação pelo teste U de Mann Whitney compreenderam a classificação dos atletas em dois grupos (baixo/médio e alto autoconceito). Já as análises de correlação entre a qualidade de vida e o autoconceito foram realizadas por meio do Coeficiente de Correlação de Spearman. O nível de significância adotado foi de 5\%. 


\section{Resultados E Discussão}

A Tabela 2, apresenta as frequências absolutas e relativas das oito dimensões dos níveis de qualidade de vida dos atletas de basquete em cadeira de rodas.

Tabela 2 - Frequências absolutas e relativas da qualidade de vida de atletas de basquete em cadeira de rodas

\begin{tabular}{cccc}
\hline \multirow{2}{*}{ Qualidade de Vida } & Regular & Boa & Muito Boa \\
\cline { 2 - 4 } & $f(\%)$ & $f(\%)$ & $f(\%)$ \\
\hline Capacidade Funcional & $4,0(7,3)$ & $17,0(30,9)$ & $34,0(61,8)$ \\
Limitação por Aspectos Físicos & $8,0(14,5)$ & $4,0(7,3)$ & $43,0(78,2)$ \\
Dor & $31,0(56,4)$ & $21,0(38,2)$ & $3,0(5,5)$ \\
Estado Geral de Saúde & $23,0(41,8)$ & $25,0(45,5)$ & $7,0(12,7)$ \\
Vitalidade & $6,0(10,9)$ & $44,0(80,0)$ & $5,0(9,1)$ \\
Aspectos Sociais & $2,0(3,6)$ & $48,0(87,3)$ & $5,0(9,1)$ \\
Limitação por aspectos emocionais & $7,0(12,7)$ & $8,0(14,5)$ & $40,0(72,7)$ \\
Saúde Mental & $1,0(1,8)$ & $38,0(69,1)$ & $16,0(29,1)$ \\
\hline
\end{tabular}

Verificou-se na Tabela 2 que os atletas consideram ter uma percepção de qualidade de vida "muito boa" nas dimensões limitação por aspectos físicos $(78,2 \%)$, limitação por aspectos emocionais $(72,7 \%)$ e capacidade funcional $(61,8 \%)$. Para a dimensão estado geral de saúde, os atletas demonstraram uma percepção de qualidade de vida de regular $(41,8 \%)$ a boa $(45,5 \%)$.

Para as dimensões aspectos sociais e vitalidade os atletas apresentaram boa percepção de qualidade de vida, com frequências de $87,3 \%$ e $80 \%$, respectivamente (TABELA 2). As percepções positivas dos atletas nestas dimensões podem decorrer do seu envolvimento com o contexto esportivo, sugerindo assim que a prática de atividade física regular contribui na melhora da percepção da qualidade de vida dos participantes. Esses achados encontram suporte nos estudos de Noce et al., (2009) que investigaram a influência da prática da atividade física na percepção da qualidade de vida de deficientes físicos. Os autores observaram que os indivíduos ativos apresentaram escores mais elevados em todas as dimensões (física, psicológica, social e ambiental) da qualidade de vida quando comparados aos deficientes insuficientemente ativos.

$\mathrm{Na}$ dimensão dor a maioria dos atletas $(56,4 \%)$ apresentou percepção regular (TABELA 2), o que pode revelar que a dor é um fator negativo para sua qualidade de vida, mesmo com a prática de atividades físicas. Este resultado vai ao encontro dos achados de Martins e Rabelo (2008) que em seu estudo buscaram comparar a percepção de qualidade de vida de 20 deficientes físicos, cadeirantes, praticantes (grupo experimental) e não-praticantes (grupo controle) de atividade física adaptada após a intervenção de um programa de exercícios físicos. Os autores identificaram que na dimensão dor não houve diferença significativa na percepção da qualidade de vida entre o grupo controle e o experimental bem como na comparação antes e após a intervenção de exercício físico. 
Além disso, a compreensão da percepção da dor nos atletas participantes do presente estudo recebe suporte dos achados de Freitas (2000) que afirma que a resposta negativa da atividade física em relação à dimensão dor pode decorrer pela postura sentada sobre a cadeira de rodas, pois as exigências de movimentos que envolvem a coluna vertebral durante a prática de atividades físicas (manobras com os membros superiores, arremessos no basquete e o manejo da cadeira) exercem maior tensão sobre a coluna vertebral (FREITAS, 2000).

As classificações dos atletas quanto ao nível de autoconceito são apresentadas na Tabela 3. Observa-se que na subescala self ético-moral $80,0 \%$ dos atletas apresentaram nível de autoconceito alto, bem como para as escalas do self somático $(54,5 \%)$, autocontrole $(54,5 \%)$, receptividade social $(52,7 \%)$ e autoconfiança $(61,8 \%)$. No geral, a maioria dos atletas ficaram classificados em autoconceito mediano ou alto.

Tabela 3 - Frequências absolutas e relativas dos níveis do autoconceito dos atletas de basquete em cadeira de rodas

\begin{tabular}{cccc}
\hline Autoconceito & Baixo f (\%) & Médio f (\%) & Alto f (\%) \\
\hline Atitude Social & $4,0(7,3)$ & $30,0(54,5)$ & $21,0(38,2)$ \\
Autoconfiança & $1,0(1,8)$ & $20,0(36,4)$ & $34,0(61,8)$ \\
Autocontrole & $2,0(3,6)$ & $23,0(41,8)$ & $30,0(54,5)$ \\
Self Ético-Moral & $2,0(3,6)$ & $9,0(16,4)$ & $44,0(80,0)$ \\
Self Somático & $3,0(5,5)$ & $22,0(40)$ & $30,0(54,5)$ \\
Receptividade Social & $2,0(3,6)$ & $24,0(43,6)$ & $29,0(52,7)$ \\
\hline
\end{tabular}

Os níveis altos na maioria das subescalas são considerados positivos para os atletas participantes deste estudo e apenas no domínio atitude social os atletas demonstraram nível médio. A subescala na qual os sujeitos apresentaram maior frequência de respostas foi a do Self Ético-Moral, a qual remete-se ao fato de as pessoas se reconhecerem como dignas e honestas, reguladas pelas normas ético-morais (TAMAYO, 1981). Segundo a mesma autora, essa subescala do autoconceito é formada pela percepção social vinda de outras pessoas e a avaliação que o indivíduo faz de si, ou seja, consiste na crença do que é bom e do que é mal para cada indivíduo. Esses achados são contrários ao estudo de Sernaglia et al., (2010) realizado com deficientes físicos praticantes de esporte adaptado nas modalidades de rúgbi e de handebol em cadeira de rodas, no qual foi constatado que os sujeitos se avaliaram de forma indiferente em relação a suas condutas e comportamentos.

Na subescala do "Self Somático" (TABELA 3) os atletas apresentaram um nível alto de autoconceito, o que pode indicar que estes se reconhecem com uma boa aparência física. Em casos de paraplegia ocorre uma alteração da imagem corporal, relacionada com a falta de sensibilidade dos membros, estando também ligada a uma desvalorização da imagem estética (TALEPOROS; MCCABE, 2001). O uso de cadeiras de rodas tem sido descrito como um fator importante que pode influenciar na percepção da imagem corporal e Mayeda (2005) destaca a importância da construção das relações físicas e psíquicas com este meio de locomoção na percepção de corpo do deficiente físico, pois o sucesso nessa relação irá 
determinar a aceitação da cadeira como extensão do corpo gerando maior satisfação com a imagem corporal.

Na Tabela 4 são apresentas as comparações das dimensões da qualidade de vida conforme a classificação sugerida do autoconceito para a subescala "autocontrole" entre os níveis baixo/médio e alto, na qual foram observadas diferenças significativas entre os grupos somente para a dimensão limitação física $(\mathrm{p}=0,029)$, na qual os atletas com alto nível de autocontrole apresentaram melhor percepção de qualidade de vida. Destaca-se que para os demais domínios do autoconceito (self ético moral, self somático, autoconfiança, atitude social e receptividade social) não foram encontradas diferenças estatisticamente significativas ( $p>0,05)$ entre os grupos baixo/médio e alto autoconceito.

Tabela 4 - Comparação das medianas (Md) da percepção da qualidade de vida dos atletas de basquete em cadeira de rodas considerando o autoconceito (dimensão autocontrole)

\begin{tabular}{cccc}
\hline Qualidade de vida & \multicolumn{2}{c}{ Autocontrole } & \multirow{2}{*}{$\boldsymbol{P}$} \\
\cline { 2 - 3 } & Baixo/Médio & Alto & \\
Capacidade Funcional & Md (Q1-Q3) & $M d(Q 1-Q 3)$ & \multirow{2}{*}{0,939} \\
Limitação física & $75.0(25,0-80,0)$ & $70,0(50,0-80,0)$ & $0,100,0)$ \\
Dor & $30,0(10,0-50,0)$ & $100,0(75,0-100,0)$ & $0,029^{*}$ \\
Estado geral de saúde & $35,0(30,0-40,0)$ & $35,0(20,0-50,0)$ & 0,316 \\
Vitalidade & $45,0(40,0-55,0)$ & $50,0(45,0-45,0)$ & 0,609 \\
Aspectos sociais & $50,0(50,0-50,0)$ & $50,0(37,0-50,0)$ & 0,214 \\
Aspectos emocionais & $68,0(33,0-100,0)$ & $100,0(68,0-10,0)$ & 0,722 \\
Saúde mental & $56,0(52,0-64,0)$ & $58,0(56,0-64,0)$ & 0,560 \\
\hline
\end{tabular}

*diferença estatisticamente significativa $(\mathrm{P} \leq 0,05)$.

Diante dos dados encontrados, percebe-se que os atletas paranaenses se percebem com um nível elevado de autoconceito, indicando que possuem disciplina sobre suas atividades. Segundo Noce et al., (2009), os benefícios psicológicos alcançados pela prática regular de atividades físicas se refletem nas relações de trabalho, na vida afetiva e social de deficientes físicos. Essa assertiva pode auxiliar na compreensão dos aspectos encontrados no presente estudo que aponta que a prática de atividade física pode refletir sobre as percepções positivas destes atletas.

Ao comparar as dimensões da qualidade de vida dos atletas conforme a classificação do autoconceito, verificou-se (Tabela 4) que houve diferença significativa para a subescala "limitação por aspectos físicos", indicando que os atletas classificados com nível alto de autocontrole se sentem menos limitados fisicamente, quando comparados aos atletas com níveis baixo/médio. Com base nestes achados, é possível entender que um bom autocontrole pode indicar que as pessoas respondem de forma emocional positiva às influências externas e isso pode refletir em sua maneira de lidar com as limitações físicas advindas da deficiência, percebendo-se menos afetadas pelas limitações.

Essas percepções positivas podem ser decorrentes das práticas esportivas e de atividades físicas que potencializam a autoconfiança e autoestima dos praticantes, 
possibilitando ao indivíduo testar suas reais capacidades e reconhecer suas limitações, adquirindo maior controle sobre suas ações e reações físicas e emocionais (GORDON, 1993). Nesse sentido, Cardoso et al. (2011) acrescentam que a prática de atividade física regular traz benefícios como a autonomia e autoconfiança, diminuindo as dificuldades enfrentadas por deficientes físicos no trabalho ou em suas casas, decorrentes da capacidade motora limitada.

Diversos estudos presentes na literatura verificaram que o bem-estar psicológico pode promover comportamentos saudáveis, uma vez que pessoas dotadas de senso de autovalor acreditam em seu poder de controle e são otimistas quanto ao futuro, além de mais propensas a adotar hábitos adequados e conscientes (RABELO; NÉRI, 2005, PEREIRA et al., 2006). Segundo Rabelo e Néri (2005), os recursos psicológicos como o otimismo, o controle pessoal e o senso de significado são importantes reservas que permitem as pessoas enfrentar melhor os eventos críticos da vida.

A Tabela 5 apresentam-se os valores da correlação entre a qualidade de vida e autoconceito dos atletas.

Tabela 5 - Correlação entre a percepção da qualidade de vida e o autoconceito de atletas de basquete em cadeira de rodas

\begin{tabular}{|c|c|c|c|c|c|c|}
\hline \multirow{2}{*}{ Qualidade de Vida } & \multicolumn{6}{|c|}{ Autoconceito } \\
\hline & 1 & 2 & 3 & 4 & 5 & 6 \\
\hline Capacidade funcional & $0,281 *$ & $0,298^{*}$ & 0,013 & 0,103 & 0,074 & 0,201 \\
\hline Limitação por aspectos físicos & 0,065 & 0,142 & 0,229 & 0,207 & 0,060 &, 053 \\
\hline Dor & $-0,094$ & $-0,113$ & 0,144 & 0,063 & $0,074-$ & 0,167 \\
\hline Estado geral de saúde & $-0,376^{* *}$ & $*-0,147$ & $-0,128$ & $-0,197$ & $0,213-$ & 0,195 \\
\hline Vitalidade & 0,024 & $-0,089$ & 0,218 & $-0,006$ & $0,035-$ & 0,128 \\
\hline Aspectos sociais & $-0,082$ & 0,169 & 0,034 & $-0,137$ & 0,010 & 0,063 \\
\hline Limitação por aspectos emocionais & is 0,193 & $0,275^{*}$ & 0,191 & 0,249 & 0,257 &, 148 \\
\hline Saúde mental & $-0,039$ & $-0,023$ & 0,085 & 0,128 & 0,072 &, 173 \\
\hline
\end{tabular}

Ao realizar os testes de correlações entre as dimensões da qualidade de vida com as subescalas do autoconceito foram verificadas correlações significativas e fracas $(\mathrm{p}<0,05)$, demonstrando uma tendência de correlação entre os aspectos psicológicos com indicadores de qualidade de vida, indicando que quanto mais elevado o autoconceito dos atletas, mais positivamente percebem sua qualidade de vida. 


\section{CONSIDERAÇÕES FINAIS}

Este estudo teve como objetivo, investigar a percepção de qualidade de vida e o nível de autoconceito em atletas da modalidade de basquetebol em cadeira de rodas, buscando especificamente comparar a percepção da qualidade de vida dos atletas considerando o autoconceito (dimensão autocontrole) e correlacionar as variáveis (qualidade de vida e autoconceito). No geral, os atletas paranaenses apresentaram boa percepção da qualidade de vida na maioria das dimensões avaliadas, exceto para os indicadores de dor, evidenciando que este é um dos fatores de maior incômodo para os atletas. Os resultados para o autoconceito indicaram níveis elevados na maioria das subescalas. Os dados encontrados no presente estudo sinalizam que a prática esportiva reflete positivamente sobre as variáveis investigadas.

Quando os grupos foram estratificados de acordo com a classificação do autoconceito (baixo/médio e alto autoconceito), foi verificado que aqueles atletas classificados com autocontrole em níveis altos apresentam uma melhor qualidade de vida para a dimensão "limitação por aspectos físicos", o que indica que aspetos psicológicos exercem influência na percepção da qualidade de vida. Foram observadas correlações significativas entre as dimensões da qualidade de vida com as subescalas do autoconceito. Nessa perspectiva, os resultados do presente estudo demonstram que a prática de paradesportos pode contribuir para elevar os níveis de qualidade de vida, bem como influenciar positivamente as esferas do comportamento psicológico, o que é essencial para a melhora da confiança e autoestima da pessoa com deficiência.

Apesar dos relevantes achados desta pesquisa, é necessário apontar algumas limitações. Primeiramente, este estudo se limitou a avaliar uma amostra de atletas paranaenses de basquetebol em cadeira de rodas do sexo masculino, o que não permite generalização dos resultados para todos os atletas da modalidade e de ambos os sexos. Além disso, o estudo foi realizado com a premissa da identificação dos fatores psicológicos (autoconceito) e percepção de qualidade de vida destes atletas, não contemplando a identificação de outras variáveis psicológicas que incidem sobre o seu bem-estar e desempenho. Nessa perspectiva, sugere-se para futuras investigações a avaliação de atletas com deficiência física de outras modalidades esportivas (coletivas e individuais), de ambos os sexos, por meio da avaliação de variáveis psicológicas como a resiliência, motivação, autoestima e necessidades psicológicas básicas.

\section{REFERÊNCIAS}

ARAÚJO, Denise Sardinha Mendes Soares; ARAÚJO, Cláudio Gil Soares. Aptidão física, saúde e qualidade de vida relacionada à saúde em adultos. Revista Brasileira de Medicina do Esporte. v. 6, n. 5, p. 194-203, 2000. Disponível em: 
<http://www.scielo.br/scielo.php?script=sci_arttext\&pid=S1517-86922000000500005>.

BRANDEN, Nathaniel. Auto-estima: como aprender a gostar de si mesmo. 35. ed. São Paulo: Saraiva, 2000.

BLAIR, Steven N. et al. Changes in physical fitness and all-cause mortality: a prospective study of healthy and unhealthy men. JAMA, v. 273, n. 14, p. 273, 1995. Disponível em: <https://jamanetwork.com/journals/jama/fullarticle/vol/273/pg/1093>.

CARDOSO, Vinícius Denardin. A reabilitação de pessoas com deficiência através do desporto adaptado. Revista Brasileira de Ciência no Esporte. Florianópolis, v. 33, n. 2, p. 529-539, 2011. Disponível em: <http://www.scielo.br/scielo.php?pid=S010132892011000200017\&script=sci_abstract\&tlng=pt $>$.

CICONELLI, Rozana Mesquita. Tradução para o português e validação do questionário genérico de avaliação de qualidade de vida. Tese (Doutorado em Ciências Médicas) Universidade Federal de São Paulo (EPM), São Paulo, 1997.

GORDON, Neil F. Stroke: Your complete exercise guide. Champaign: Human Kinetics, 1993.

FREITAS, Patricia Silvestre. Manejo em cadeira de rodas. In: FREITAS, Patricia Silvestre (Org). Educação Física e Esporte para Deficientes: coletânea. Uberlândia.UFU, 2000.

GALLAHUE, David; OZMUN, John. Compreendendo o desenvolvimento motor: bebês, crianças, adolescentes e adultos. São Paulo: Phorte, 2001.

INSTITUTO BRASILEIRO DOS DIREITOS DA PESSOA COM DEFICIÊNCIA. Inclusão social da pessoa com deficiência: medidas que fazem a diferença. Rio de Janeiro: IBDD, 2008. Disponível em: <http://www.ibdd.org.br/arquivos/inclusaosocial.pdf>. Acesso em: 03 mar. 2017.

MADUREIRA, Alberto Saturno; MAIA, Maria de Fátima. M; FONSECA, Silvio Aparecido. Estilo de vida e atividade física habitual de professores de educação física. Revista Brasileira de Cineantropometria \& Desenpenho Humano, v. 5, n. 1, p. 54-62, 2003. Disponível em: $<$ http://cev.org.br/biblioteca/estilo-vida-atividade-fisica-habitual-professores-educacaofisica/>.

MARTINS, Débora Lourdes; RABELO, Ricardo José. Influência da atividade física adaptada na qualidade de vida de deficientes físicos. Revista Movimentum, v. 3, n. 2, 2008. Disponível em:

<https://www.unilestemg.br/movimentum/Artigos_V3N2_em_pdf/movimentum_v3_n2_mart ins_debora_rabelo_ricardo_2007.pdf>.

MAYEDA, Silvia. Minha cadeira de rodas, meu corpo: as diferenças percepções corporais dos usuários. 2005. 58 f. Monografia (Especialização) - Universidade Estadual de Campinas, Campinas, 2005.

NOCE, Franco et al. A percepção de qualidade de vida de pessoas portadores de deficiência física pode ser influenciada pela prática de atividade física. Revista Brasileira de Medicina do 
Esporte, v. 15, n. 3, p. 174-178, 2009. Disponível em:

$<$ http://www.scielo.br/scielo.php?pid=S1517-

$86922009000300002 \&$ script=sci_abstract\&tlng=pt $>$.

PAFFENBARGER JUNIOR, Ralph S. J. Forty years of progress: physical activity, health, and fitness. In: AMERICAN College of Sports Medicine 40th Anniversary Lectures.

Madison: ACSM, 1994.

PEREIRA, Renata Junqueira et al. Contribuição dos domínios físico, social, psicológico e ambiental para a qualidade de vida global de idosos. Revista de Psiquiatria, v. 28, n. 1, p. $27-$ 38, 2006. Disponível em: <http://www.scielo.br/scielo.php?script=sci_arttext\&pid=S0101$81082006000100005>$.

RABELO, Dóris Firmino; NERI, Anita Liberalesso. Recursos psicológicos e ajustamento pessoal frente a incapacidade funcional na velhice. Psicologia em Estududo, Maringá, v. 10, n. 3, p. 403-412, 2005. Disponível em: <http://www.scielo.br/scielo.php?pid=S1413$73722005000300008 \&$ script $=$ sci_abstract\&tlng=pt $>$.

SERNAGLIA, Mirella Bagdadi et al. Avaliação do autoconceito em cadeirantes praticantes de esporte adaptado. Pensar a Prática, Goiânia, v. 13, n. 3, p. 118, 2010. Disponível em: <https://www.revistas.ufg.br/fef/article/view/10223>.

TALEPOROS, Geoge; MCCABE, Marita. The impact of physical disability on body esteem. Sexuality and disability, v. 19, n. 4, p. 293-308, 2001. Disponível em:

<https://link.springer.com/article/10.1023/A:1017909526508>.

TAMAYO, Alvaro. EFA: Escala Fatorial de Autoconceito. Arquivo Brasileiro de Psicologia, v. 33, n. 4, p. 87-102, 1981. Disponível em:

<http://bibliotecadigital.fgv.br/ojs/index.php/abp/article/view/18617>.

TAMAYO, Alvaro. Autoconcepto y prevención. In: ROJAS, J. R. (Ed.). Quinta antología: salud y adolescência. San José de Costarica: Caja Costarricense de Seguro Social, 1993. p. $18-28$.

TAMAYO, Alvaro et al. A influência da atividade física regular sobre o autoconceito. Estudos de psicologia, Natal, v. 6, n. 2, p. 157-165, 2001. Disponível em:

<http://www.scielo.br/scielo.php?pid=s1413-

294x2001000200004\&script=sci_abstract\&tlng=pt>.

ZUCHETTO, Ângela Teresinha; CASTRO, Rosângela Laura Ventura Gomes. As Contribuições das Atividades Físicas para a Qualidade de Vida dos Deficientes Físicos. Revista Kinesis, n. 26. p. 26-52. 2002. Disponível em:

<https://periodicos.ufsm.br/kinesis/article/view/8010>.

LATORRE-ROMÁN, Pedro Ángel; MARTÍNEZ, Ana Vanessa Navarro, GARCÍAPINILLOS, Felipe. Effect of a physical activity program on sport enjoyment, physical activity participation, physical self-concept and quality of life in children with asthma. Motriz: Revista de Educação Física, v. 21 n. 4, p. 386-392, 2015. Disponível em: <http://www.scielo.br/scielo.php?script=sci_arttext\&pid=S1980-65742015000400386>. 
MARTINS, Raquel de Melo; DASCAL, Juliana Bayeux; MARQUES, Inara. Equilíbrio postural em idosos praticantes de hidroginástica e karatê. Revista Brasileira de Geriatria e Gerontologia, Rio de Janeiro, v. 16, n. 1, p. 61-69, 2013. Disponível em:

$<$ http://www.scielo.br/scielo.php?pid=S1809-

$98232013000100007 \&$ script $=$ sci_abstract\&tlng $=$ pt $>$.

OLIVEIRA, Vinícius Machado et al. Aptidão funcional de mulheres de meia-idade e idosas ingressantes em um programa de atividades físicas em unidades de saúde. Revista Brasileira de Qualidade de Vida, Ponta Grossa, v. 8, n. 1, p. 85-100, 2016. Disponível em: $<$ https://periodicos.utfpr.edu.br/rbqv/article/view/3690>. 